Case Report

\title{
Restrictive Cardiomyopathy Revealing Systemic Sclerodermia
}

\author{
Soukaina Safir", Rim Ouazzane, Amal Moukhliss, Hanane Choukrani, Abdenacer Drighil, \\ Leila Azzouzi, Rachida Habbal
}

Department of Cardiology, Ibn Rochd University Hospital, Casablanca, Morocco

\author{
Email address: \\ sknsafir@gmail.com (S. Safir) \\ ${ }^{*}$ Corresponding author
}

\section{To cite this article:}

Soukaina Safir, Rim Ouazzane, Amal Moukhliss, Hanane Choukrani, Abdenacer Drighil, Leila Azzouzi, Rachida Habbal. Restrictive Cardiomyopathy Revealing Systemic Sclerodermia. American Journal of Internal Medicine. Vol. 8, No. 2, 2020, pp. 62-65.

doi: 10.11648/j.ajim.20200802.13

Received: January 14, 2020; Accepted: February 6, 2020; Published: March 10, 2020

\begin{abstract}
Primary myocardial involvement is common in systemic sclerosis. There is growing evidence strongly suggesting that this involvement is linked to repeated focal ischemic lesions causing irreversible myocardial fibrosis. It can affect all heart structures. We report the case of a 65 years old male patient, without any personal medical history, admitted for inaugural right heart decompensation. The diagnosis of restrictive cardiomyopathy is based mainly on the following criteria: on echocardiography, hypertrohy of the right ventricle with alteration of the longitudinal systolic function, dilation of the right atrium, with good overall contractility. On right cardiac catheterization: elevation and equalization of the telesystolic pressures of the right atrium, right ventricle, and pulmonary artery with appearance of dip plateau on the right ventricle curve. Scleroderma was evoked considering following statements: the presence of Raynaud's phenomenon, unknown by the patient (presence of dermal sclerosis with pericapillary oedema and capillary dystrophy on capillaroscopy), cutaneous involvement (presence of perivascular and perineural mononuclear infiltrates, with collagen fibers increased in number and thickness), pulmonary involvement (diffuse bilateral interstitial lung disease) and renal involvement (moderately impaired function and positive proteinuria). The concept of cardiac dysfunction in scleroderma and other rheumatologic conditions has received new interest with the advent of newer non-invasive imaging techniques. Therfore, it would be necessary to search the cardiac involvement especially subclinical one in this type of system disease, as well as to confirm the systemic origin in front of certain forms of cardiopathies in particular restrictive cardiomyopathy.
\end{abstract}

Keywords: Systemic Sclerodermia, Restrictive Cardiomyopathy, Echocardiography, Right Cardiac Catheterization

\section{Introduction}

Primary myocardial involvement is common in systemic sclerosis. Increasing evidence strongly suggests that this involvement is related to repeated focal ischemic injury causing irreversible myocardial fibrosis. Heart disease in systemic scleroderma is initially often asymptomatic, but it is necessary to screen for it systematically as it has a poor prognosis. Its incidence is estimated between $15 \%$ and $35 \%$ and can affect all cardiac structures. It can manifest as pericarditis, rhythm or conduction disorder, valve damage, ischemic or hypertrophic myocardial disease and heart failure. High-speed heart failure is not classically described in scleroderma, but rather the prerogative of hyperthyroidism, anemia, Paget's disease, arteriovenous fistulas or vitamin B1 deficiency.

\section{Case Report}

We report the case of a 65 years old patient, admitted for inaugural right heart decompensation. In cardiovascular risk factors, active smoking for 40 years, age, and sedentary lifestyle. The patient did not have any personal medical none surgical history. In the family history, there is a sudden death in a first degree cousin, at the age of 24 years old. The initial 
symptomatology started 7 months ago by the installation of progressive dyspnea with general asthenia. The symptoms worsened one month before hospitalization due to excessive salt intake. The patient presented with worsening dyspnea, palpitation and lower limb edema.

At hospitalization, the patient was hemodynamically and respiratory stable; blood pressure at $900 / 600 \mathrm{MmHg}$, pulse rate $100 / \mathrm{min}$. He presented with turjescence of the jugular veins, hepatojugular reflux, and hepatomegaly at $15 \mathrm{~mm}$. The pleuropulmonary examination found a symmetrical thorax, with a vesicular murmur well perceived, and vocal vibrations well transmitted, without rattles. Cutaneous examination revealed the presence of purpuric stains in the face and the hands, that did not disappear in vitro pressure. The rest of the clinical exam was normal. On his electrocardiogram, there was a regular sinus rhythm at $75 / \mathrm{min}$, fixed PR at $200 \mathrm{~ms}$, with a complete right limb block and secondary repolarization disorders.
On his echocardiography, a non dilated, non-hypertrophied left ventricule, with paradoxal septum, with good global contractility. $\mathrm{LVEF}=55 \% \mathrm{SBP}$. The mitral profile was a relaxation anomaly with low left ventricular filling pressures. Dilated right atrium (SOD $39 \mathrm{~cm}^{2}$ ). Minimal mitral and aortic regurgitation. The right ventricle was dilated, hypertrophied, with longitudinal systolic dysfunction (TAPSE at $13 \mathrm{~mm}$, SDV at $7 \mathrm{~cm} / \mathrm{s}$ ). Laminar tricuspid regurgitation with coaptation defect of tricuspid valves that are infiltrated. Inferior veina cava was dilated and non-compliant. Minimal Pericardial effusion toward the right cavities.

In right catheterization, we have elevation and equalization of the telesystolic pressures of the OD, VD, and AP with appearance of dip plateau on the right ventricule curve. Moderate pulmonary hypertension (PAPm at $32 \mathrm{mmhG}$ ). (figure 1)

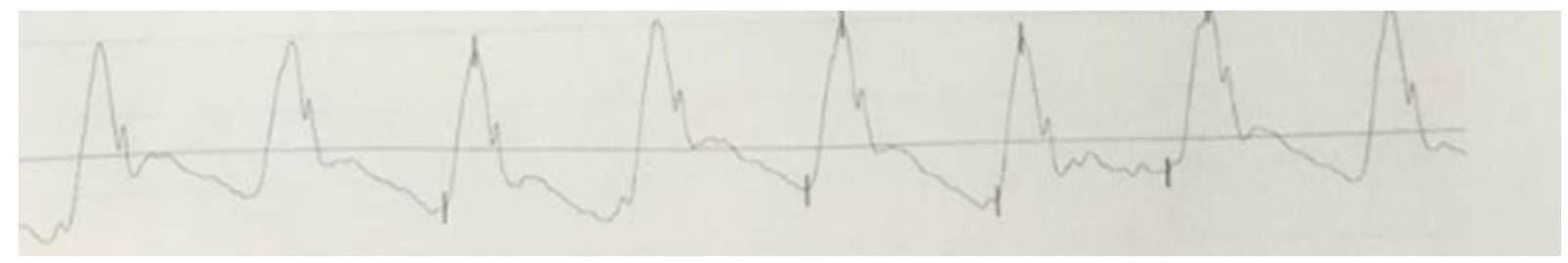

Figure 1. Right catheterization showing dipplateau on the pulmonary artery curve.

The chest CT angiography showed cardiomegaly with pericardial effusion, dilation of pulmonary artery trunk and right and left pulmonary arteries, and diffuse bilateral interstitial lung disease. (Figure 2)

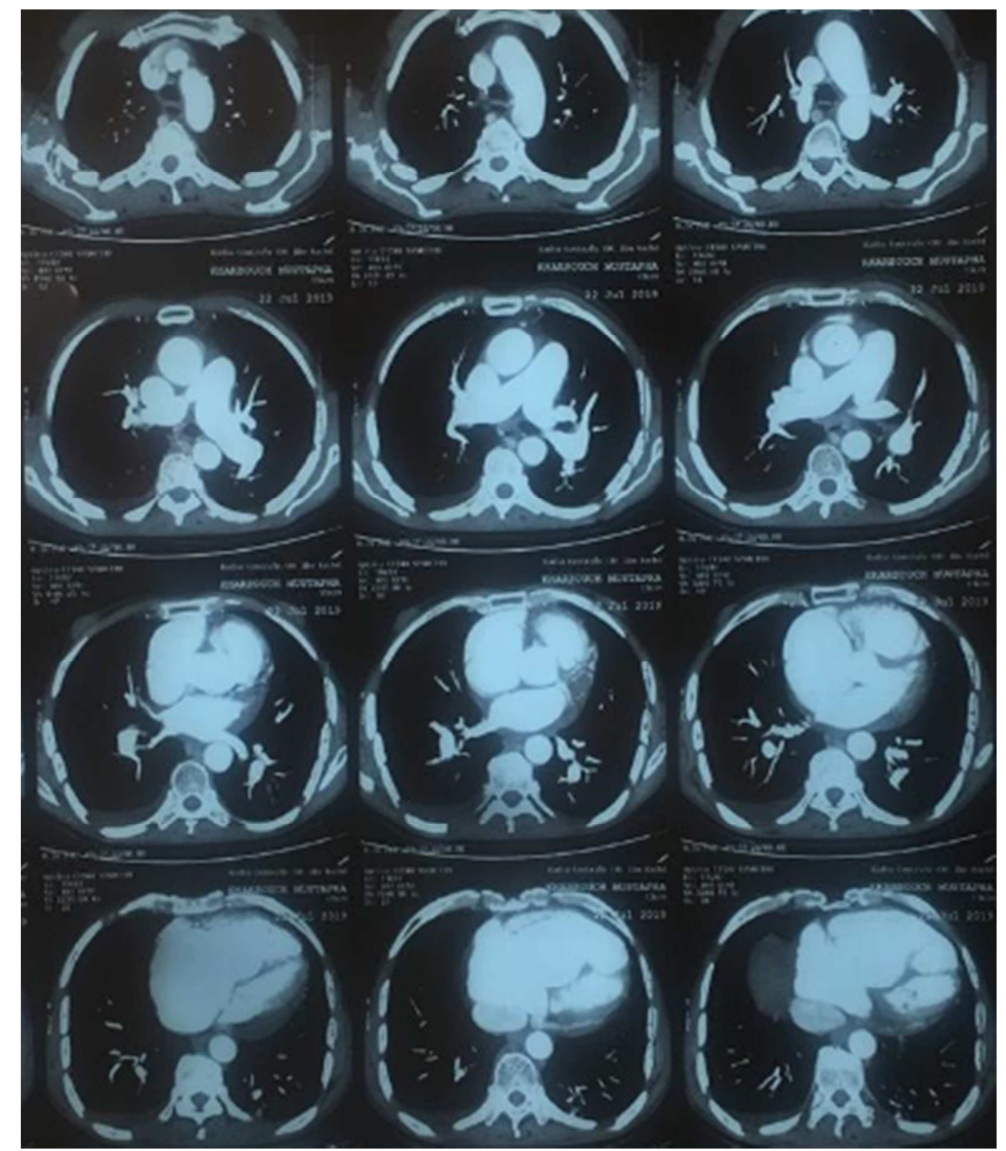

Figure 2. Chest CT angiography showing cardiomegaly with pericardialeffusion, dilation of pulmonary artery trunk. 
In the biological assessment, the renal function was moderately impaired, with a DFG at $39 \mathrm{ml} / \mathrm{min}$, positive proteinuria at $400 \mathrm{mg} / 24 \mathrm{~h}$. Converting enzyme rate was low. Immunological assessment was negative. Erythrocyte sedimentation ratewas high. Salivary biopsy showed lymphocytic inflammation of the salivary glands. Histopathological examination of a cutaneous biopsy demonstrated the presence of perivascular and perineural mononuclear infiltrates, with collagen fibers increased in number and thickness. Capillaroscopy demonstrated the presence of dermal sclerosis with pericapillary oedema and capillary dystrophy. Thyroid assessment was normal. Tumor markers were normal. Oesophageal fibroscopy demonstrated non-specific esophagitis. Hands $\mathrm{X}$ ray showed a diffuse demineralization, without acro-osteolysis, or articular narrowing.

Scleroderma was evoked considering following statements: the presence of Raynaud's phenomenon (unknown by the patient), cutaneous involvement, pulmonary involvement and renal involvement.

The patient was put on cyclophosphamid infusion, associated to diuretic and supplementation $\mathrm{KCL}$, Betablockers.

Our case report showed that systemic sclerodermia can be presented with a congestive heart failure with or without any other clinical symptoms or systemic sclerodermia. Therefore it is necessary to think about this disease mainly in young women with progressive dyspnea and with an echocardiographic with signs of restrictive cardiomyopathy.

\section{Discussion}

Systemic Sclerosis (SSc) is a disease characterized by abnormalities in the functioning of small blood vessels as well as the immune system, ultimately leading to excessive fibrosis of the skin and organs, such as the lungs, digestive tract, kidneys and heart.

Cardiac manifestations are common in patients with SSc (approximately $15 \%$ to $35 \%$ of patients) and occur in both the limited and diffuse form of the disease. However, heart disease can be silent in some people, causing few signs and symptoms. When heart problems cause more obvious symptoms, the progression is often more severe.

The prevalence of primary cardiac involvement in SSc is difficult to determine given variable clinical presentations of cardiac manifestations, applied diagnostic tools and diverse patient populations. It should be noted that the results of histological studies, which frequently reveal the presence of myocardial involvement, often disagree with those of clinical studies, carried out with different evaluation techniques [1]. Cardiac involvement can be manifested by pericardial disease, conduction system abnormalities, arrythmias and myocardial disease.

Clinical examination and routine non-invasive investigations, such as electrocardiogram and thoracic X-ray, are applied in the everyday cardiac assessment, but their sensitivity is low [2-4].

Signs for cardiac involvement have been detected with a prevalence of $15 \%$ in a cohort of 953 patients with diffuse cutaneous SSc based on clinical findings, echocardiography, electrocardiography, or Holter monitoring [5].

Conventional echocardiography is used for cardiac assessment in most studies. Depressed left ventricle (LV) contractility has been reported in only a few patients, whereas up to $40 \%$ present with relaxation abnormalities, valvular regurgitation and possible right ventricular (RV) pathology [1].

LV systolic dysfunction is among the rarest findings in SSc patients. In a large multi-centered study, which included 570 SSc patients, the prevalence of LV systolic dysfunction was found to be $1.4 \%$, whereas LV hypertrophy and LV diastolic dysfunction were observed in $22.6 \%$ and $17.7 \%$ of patients, respectively [6]. In a recent, large European League Against Rheumatism Scleroderma Trials and Research study (including 7073 consecutive SSc patients with a mean age of $56 \pm 14$ years) the prevalence of reduced LV ejection fraction was found to be $5.4 \%$ [7].

Cardiac MRI detected heart pathologies in up to $75 \%$ (39/52) of cases, including increased intensity signal of the myocardium in $\mathrm{T} 2$, thinning of the $\mathrm{LV}$, pericardial effusion, reduced LV and RV ejection fractions, LV diastolic dysfunction and kinetic abnormalities, and myocardial delayed contrast enhancement [8].

The main clinical consequence of myocardial lesions is diastolic LV dysfunction, and less frequently systolic dysfunction, which both may be asymptomatic. In addition, different forms of atrial and ventricular arrhythmias, as well as symptomatic heart failure, may occur $[2,9]$.

Cardiac catheterization is indicated in SSc for diagnosis of PAH, constrictive pericarditis, cardiac tamponade and epicardial coronary artery disease, for performing endomyocardial biopsy in cases of suspected infiltrative cardiac disease [2].

During RHC, vasodilator testing is performed in order to predict the therapeutic response. The response is defined as a reduction $\geq 10 \mathrm{mmHg}$ to a mean $\mathrm{PAP} \leq 40 \mathrm{mmHg}$, without a decrease in cardiac output [10].

Verapamil should be avoided because of its potential for negative inotropic effects. High doses of calcium-channel blockers may improve survival in patients with primary PAH who respond with reductions in pulmonary arterial pressure and vascular resistance [11].

Early diagnosis of SSc-PAH and early subsequent intervention are essential for delaying disease progression. Early detection of $\mathrm{PAH}$, when patients have few or no symptoms (i.e., functional class I and II), is challenging. Available data broadly support annual screening of all SSc patients with and without symptoms. Patients with SSc who are at high risk for development of $\mathrm{PAH}$ are those with $\mathrm{DL}_{\mathrm{CO}}<$ $60 \%$ predicted or who have declining $\mathrm{DL}_{\mathrm{CO}}$ (e.g., $20 \%$ decrease over a one-year period). Doppler echocardiography conducted at rest is considered to be the method of choice for 
PAH screening. For patients with TRV $>3.4 \mathrm{~m} / \mathrm{s}$ (corresponding to a systolic PAP $>50 \mathrm{mmHg}$ ) or with a TRV between 2.9 and $3.4 \mathrm{~m} / \mathrm{s}$ (corresponding to a systolic PAP between 34 and $49 \mathrm{mmHg}$ ) in the presence of other signs suggestive of PAH, noninvasive workup is recommended, including biomarkers, high-resolution CT and decision for confirmation of PAH via RHC $[12,13]$.

Key points:

1. Subclinical cardiac involvement is frequent in systemic sclerosis, the clinical significance of which is not presently known.

2. Clinically apparent cardiac involvement in systemic sclerosis is found in upto $15 \%$ of patients and is associated with an adverse outcome.

3. The pathophysiology of cardiac involvement in systemic sclerosis is unknown. Cardiac Raynauld's phenomenon, accelerated coronary atherosclerosis, and autoimmune myocardial damage may all contribute.

4. Newer investigation techniques which may help identify patients with active cardiac involvement include Troponin testing and Cardiac MRI scanning.

\section{Conclusion}

The concept of cardiac dysfunction in scleroderma and other rheumatologic conditions has received new interest with the advent of newer non-invasive imaging techniques as well as the interest in detecting subclinical disease. With this increased interest in cardiac manifestations in scleroderma comes the fact that long term studies are needed to better assess the appropriate screening and treatment in this patient population. When clinically manifested, cardiac involvement is thought to be an important prognosis factor.

\section{Conflict of Interest Statement}

There is no conflict of interest for this case report.

\section{Acknowledgements}

We gratefully acknowedge all the participants who accepted to take part in this study.

\section{References}

[1] Allanore Y, Avouac J, Kahan A. Systemic sclerosis: an update in 2008. Joint Bone Spine. 2008; 75: 650-655.

[2] Varga J, Denton CP, Wigley FM. Scleroderma. New York: Springer; 2012. pp. 361-371; 373-395.
[3] Ferri C, Emdin M, Nielsen H, Bruhlmann P. Assessment of heart involvement. Clin Exp Rheumatol. 2003; 21: S24-S28.

[4] Kahan A, Allanore Y. Primary myocardial involvement in systemic sclerosis. Rheumatology (Oxford) 2006; 45 Suppl 4: iv14-iv17.

[5] Steen VD, Medsger TA. Severe organ involvement in systemic sclerosis with diffuse scleroderma. Arthritis Rheum. 2000; 43: 2437-2444.

[6] Groote P, Gressin V, Hachulla E, Carpentier P, Guillevin L, Kahan A, Cabane J, Francès C, Lamblin N, Diot E, Patat F, Sibilia J, Petit H, Cracowski JL, Clerson P, Humbert M, ItinerAIR-Scleroderma Investigators. Evaluation of cardiac abnormalities by Doppler echocardiography in a large nationwide multicentric cohort of patients with systemic sclerosis. Ann Rheum Dis. 2008 Jan; 67 (1): 31-6.

[7] Allanore Y, Meune C, Vonk MC, Airo P, Hachulla E, Caramaschi P, Riemekasten G, Cozzi F, Beretta L, Derk CT, Komócsi A, Farge D, Balbir A, Riccieri V, Distler O, Chialà A, Del Papa N, Simic KP, Ghio M, Stamenkovic B, Rednic S, Host $\mathrm{N}$, Pellerito R, Zegers E, Kahan A, Walker UA, Matucci-Cerinic M, EUSTAR co-authors. Prevalence and factors associated with left ventricular dysfunction in the EULAR Scleroderma Trial and Research group (EUSTAR) database of patients with systemic sclerosis. Ann Rheum Dis. 2010 Jan; 69 (1): 218-21.

[8] Hachulla AL, Launay D, Gaxotte V, de Groote P, Lamblin N, Devos P, Hatron PY, Beregi JP, Hachulla E. Cardiac magnetic resonance imaging in systemic sclerosis: a cross-sectional observational study of 52 patients. Ann Rheum Dis. 2009 Dec; 68 (12): 1878-84.

[9] Ferri C, Giuggioli D, Sebastiani M, Colaci M, Emdin M. Heart involvement and systemic sclerosis. Lupus. 2005; 14: 702707.

[10] McLaughlin VV, Archer SL, Badesch DB, Barst RJ, Farber HW, Lindner JR, Mathier MA, McGoon MD, Park MH, Rosenson RS, et al. ACCF/AHA 2009 expert consensus document on pulmonary hypertension a report of the American College of Cardiology Foundation Task Force on Expert Consensus Documents and the American Heart Association developed in collaboration with the American College of Chest Physicians; American Thoracic Society, Inc.; and the Pulmonary Hypertension Association. J Am Coll Cardiol. 2009; 53: 1573-1619.

[11] Rich S, Kaufmann E, Levy PS. The effect of high doses of calcium-channel blockers on survival in primary pulmonary hypertension. N Engl J Med. 1992; 327: 76-81.

[12] Vachiéry JL, Coghlan G. Screening for pulmonary arterial hypertension in systemic sclerosis. Eur Respir Rev. 2009; 18: 162-169.

[13] Schwaiger JP, Khanna D, Gerry Coghlan J. Screening patients with scleroderma for pulmonary arterial hypertension and implications for other at-risk populations. Eur Respir Rev. 2013; 22: $515-525$. 